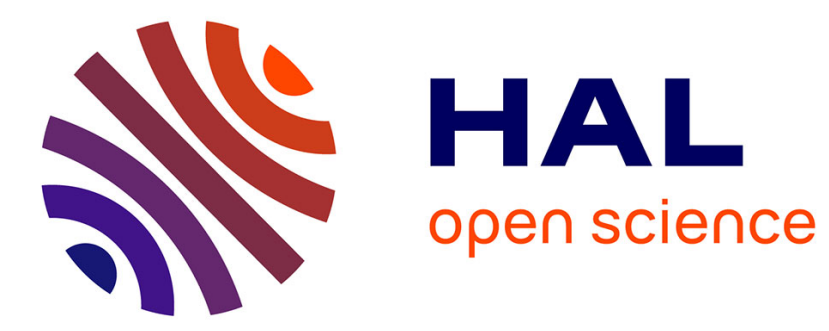

\title{
Toward a Robust Motorcycle Braking
}

Mohammed El-Habib Dabladji, Dalil Ichalal, Hichem Arioui, Saïd Mammar

\section{To cite this version:}

Mohammed El-Habib Dabladji, Dalil Ichalal, Hichem Arioui, Saïd Mammar. Toward a Robust Motorcycle Braking. IEEE Transactions on Control Systems Technology, 2017, 25 (3), pp.1052-1059. 10.1109/TCST.2016.2591329 . hal-01352676

\section{HAL Id: hal-01352676 https://hal.science/hal-01352676}

Submitted on 19 Dec 2019

HAL is a multi-disciplinary open access archive for the deposit and dissemination of scientific research documents, whether they are published or not. The documents may come from teaching and research institutions in France or abroad, or from public or private research centers.
L'archive ouverte pluridisciplinaire HAL, est destinée au dépôt et à la diffusion de documents scientifiques de niveau recherche, publiés ou non, émanant des établissements d'enseignement et de recherche français ou étrangers, des laboratoires publics ou privés. 


\title{
Towards a Robust Motorcycle Braking
}

Mohammed El-Habib Dabladji, Dalil Ichalal, Hichem Arioui and Saïd Mammar Senior member, IEEE

\begin{abstract}
In this paper, a robust braking controller is proposed for motorcycles. Its aim is to control both longitudinal slips of the vehicle to optimal ones using a sliding mode controller. The robustness of this controller is proved with respect to change of road adherence, load transfer, tires characteristics and lateral movements. The optimal target slip is computed thanks to an algorithm inspired from the Maximum Power Point Tracking (MPPT) methods. The objective of this algorithm is to seek the appropriate longitudinal braking forces and to avoid rear wheel's loss of contact. Simulations on a multibody simulator are given to enhance the performances of the controller in different scenarios.
\end{abstract}

Index Terms

Motorcycles, longitudinal slip controller, sliding mode controller , MPPT

\section{INTRODUCTION}

Riders of motorcycles are considered among the most vulnerable drivers. In France, riders of powered twowheeled vehicles are present in only $1.9 \%$ of the road traffic but at the same time, they are involved in $24 \%$ of fatal accidents. In this context, it is essential to develop appropriate safety systems for motorcycles. This includes preventive safety systems such as airbags, active safety systems like braking and traction control assist [1] and observers for motorcycle's dynamics [2]. The hard braking is an important issue and is often blamed as a source of accidents for motorcycles. The problem of braking is even more dangerous when road adherence becomes insufficient.

For wheel slip control, several commercial devices already exist such as anti-lock braking systems (ABS) for standard cars [3]. Wheel slip control have been developed thanks to technological advancement in hydraulic braking actuators which allow a continuous modulation of the braking torque. In this context, many approaches have been proposed to control wheel slip: a sliding mode controller in [4], a fuzzy controller in [5] and LQR scheduling gain controllers in [6], [7]. However, all these works do not take into account uncertainties in the tire-road friction forces, variations in road adherence and/or lateral dynamics.

To overcome to this problem, robust controllers seem to be good solutions. In [8], a nonlinear output controller is proposed but its main drawback is the generation of a limit cycle for some set-points longitudinal slips. In [9], a

The authors are with the Informatics, Integrative Biology and Complex Systems Laboratory (IBISC EA-4526), Université d'Évry Val d'Essonne, 91020 Évry, France. 
nonlinear cascaded feedback and feedforward algorithm is presented. However, the vehicle was considered braking on a straight line and the vertical forces are considered constant. In [10], authors use a second order sliding mode controller for the traction control for motorcycles. However, they do not study the robustness to lateral solicitations and the controller's gains depend on several unknown bounded functions which depend on the engine torque.

Moreover, in the above papers, the optimal target slip is considered known and constant which is not really true because it varies with respect to pneumatic parameters and road characteristics. This remains an open problem, but few papers have presented some ideas [11]. This problem is even more difficult in the case of motorcycle where load transfer and risk of tire's loss of contact should be considered more seriously. In [12], a solution was presented to prevent tire's loss of contact. An algorithm was used to switch between a slip controller and a load controller. However, the optimal slip for the controller was considered constant and known. Another solution was proposed in [13] to take into account lateral dynamics but with the assumption of knowledge of current road condition, and the optimization is done off-line.

The contributions of this paper are:

- The control of front and rear longitudinal slips using sliding mode techniques. The proposed controller is robust to load transfer, tire characteristics and lateral dynamics. Moreover, the tire force model is no more needed. The controller can be applied for the control of front or rear wheels separately and can also be applied for the control of both wheels.

- An on-line algorithm is given to find the best longitudinal slip that maximizes the longitudinal braking forces and avoid rear wheel's loss of contact. In this work, longitudinal acceleration is used by the algorithm and front and rear slips are controlled to the same target slip. However, if longitudinal forces are measured or estimated [14], the algorithm may be modified in order to control each slip to its optimal value. For brevity, only the first case is considered in this paper.

\section{PROBLEM FORMULATION}

throughout the paper, The important variables to be used are defined in the Table I. For brevity, the index $i$ for a variable $z_{i}$ refers to both front $(i=f)$ and rear $(i=r)$ wheels' variables. For example: $z_{i}=h\left(x_{i}, y_{i}\right)$ means that $:\left\{\begin{array}{l}z_{f}=h\left(x_{f}, y_{f}\right) \\ z_{r}=h\left(x_{r}, y_{r}\right)\end{array}\right.$

The main contribution of this work is the algorithm to find the longitudinal slip guaranteeing maximum desired deceleration. First the influence of longitudinal slip on the longitudinal forces is examined on figure 1.

This figure is obtained for a straight-line braking scenario and for a suitable road adherence. The longitudinal forces and the wheel slip are negatives. Note that there exists a longitudinal slip of $\lambda \approx-0.12$ which gives the maximum longitudinal force. So, to obtain the maximum deceleration, the longitudinal slip must be controlled to this peak $\lambda^{*}$. However, this optimal target slip varies with respect to the vertical forces, the road adherence, the tire characteristics and the lateral dynamics. Using the tire and the vehicle characteristics to find analytically this optimal longitudinal slip seems to be impossible. This is why an innovative method is proposed in this paper to find empirically this optimal slip. 
TABLE I

PARAMETERS' DEFINITION

\begin{tabular}{ll}
\hline Parameters & Definition \\
\hline$B_{f}, B_{r}$ & braking torques applied to the front and the rear wheel \\
$v_{x}, a_{x}$ & longitudinal velocity and acceleration \\
$F_{x f}, F_{x r}$ & front and rear longitudinal forces \\
$F_{z f}, F_{z r}$ & front and rear vertical forces \\
$\omega_{f}, \omega_{r}$ & front and rear wheel angular speeds \\
$R_{f}, R_{r}$ & front and rear tire radii \\
$i_{f y}, i_{r y}$ & front and rear rotational inertia of wheels \\
$M=M_{m}+M_{r}$ & sum of the motorcycle and rider masses \\
$\lambda_{f}, \lambda_{r}$ & front and rear longitudinal slips \\
$\beta$ & road adherence \\
$\alpha_{f}, \alpha_{r}$ & front and rear lateral slip angles \\
\hline
\end{tabular}

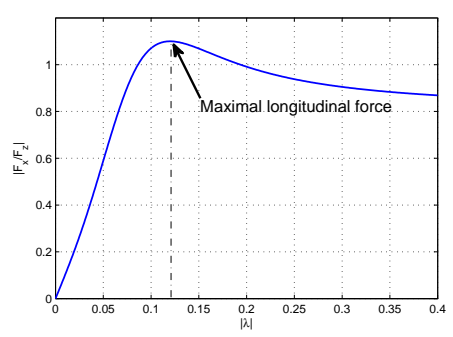

Fig. 1. Plot of the longitudinal friction coefficient as function of the longitudinal wheel slip

Another critical phenomena can be observed for motorcycles, which makes the optimal braking more difficult than four-wheeled vehicles. For example, for a given motorcycle on a dry asphalt, an adequate braking control law is used to control the front longitudinal slip to $\lambda^{*}=-0.09$. The results of simulation on the BikeSim simulator are given in figure 2. At $t=3.5 \mathrm{~s}$, it can be seen that because of the load transfer, the rear vertical force vanishes. This will cause loss of rear tire's contact to the ground and the forward flip over of the motorcycle, called stoppie. This phenomenon is even more important when the road adherence is suitable (dry surfaces) because it is easier to reach large decelerations.

According to [15], the critical deceleration that must not be exceeded depends on the position of the center of gravity of the motorcycle, its longitudinal velocity and its aerodynamics characteristics. It is given by the equation:

$$
\frac{-a_{x}}{g} \leq \frac{l_{f}}{h}+\frac{C_{d} v_{x}^{2}}{M g}
$$

where $C_{d}$ is the drag aerodynamic coefficient and the others parameters are defined above.

Finally, it is concluded that longitudinal forces have a peak value with respect to the longitudinal slip which guarantees the maximal deceleration. If the road adherence is precarious, there is no risk to reach the critical flip 

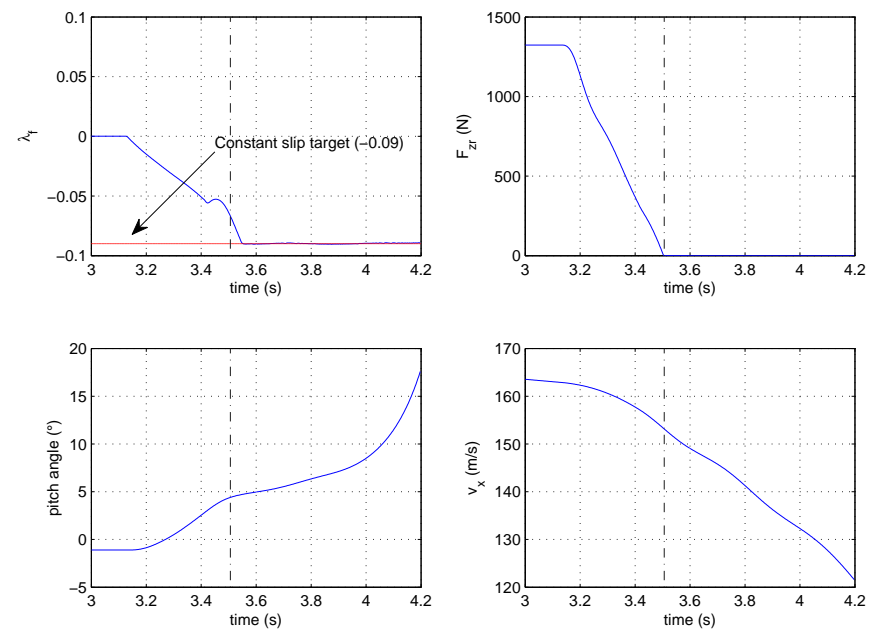

Fig. 2. Braking maneuver at constant target slip $\lambda_{f}^{*}=-0.09$. Top left: front slip. Top right: rear vertical force. Bottom left: pitch angle. Bottom right: longitudinal velocity.

over deceleration limit and the optimal braking involves the control of the longitudinal slip to the optimal one. If the road adherence is suitable (dry surfaces), the critical deceleration may easily be reached and it is useless and even dangerous to try attempting the optimal longitudinal slip.

In this paper, the problem of optimal braking is discussed as follows. In section III, a model for the motorcycle's tires and braking system is described. Section IV is devoted to the design of the controller and section V to the algorithm used in the search of the optimal longitudinal slip. Section VI assesses the effectiveness of the proposed controller and algorithm with different simulation scenarios on a multibody simulator (BikeSim).

\section{MOTORCYCLE TIRES AND BRAKING SYSTEM MODELLING}

In this section, the equations describing the tires and braking systems are given. Even simple, the proposed model will take into account all the important aspects of the vehicle with respect to the longitudinal dynamics.

\section{A. Wheels dynamics}

For the wheels' dynamics, the following assumptions are considered:

\section{Assumption 1:}

- a hydraulic braking actuator is considered, but the fluid dynamics are neglected. So, the relation between the braking torque and the braking fluid pressure is linear. However, the braking actuator dynamics will be taken into account in the validation phase ;

- the suspension dynamics are neglected.

One of the objectives of this work is to control front and rear longitudinal slips of a motorcycle to a reference one under braking. So, only the braking torques are considered as external moments for wheels' dynamics. Thus, 
the wheels' rotational model is given by the set of equations (2).

$$
\left\{\begin{array}{ccc}
I_{y i} \frac{d \omega_{i}}{d t} & = & -R_{i} F_{x i}+B_{i} \\
M a_{x} & = & F_{x f}+F_{x r}-C_{d} v_{x}^{2} \\
\lambda_{i} & = & \frac{R_{i} \omega_{i}-v_{x}}{\max \left\{v_{x}, R_{i} \omega_{i}\right\}}
\end{array}\right.
$$

$B_{i}$ is the braking torque and is always negative. When braking, $v_{x}>R_{i} \omega_{i}$ and the longitudinal slip is governed by: $\lambda_{i}=\frac{R_{i} \omega_{i}-v_{x}}{v_{x}}$.

Let us consider the following state vector:

$$
\left(\begin{array}{c}
x_{1 i} \\
x_{2 i}
\end{array}\right)=\left(\begin{array}{c}
\lambda \\
R_{i} \frac{d \omega_{i}}{d t}-a_{x}
\end{array}\right)
$$

with $a_{x}=\frac{d v_{x}}{d t}$. The following state space representation is obtained:

$$
\left\{\begin{aligned}
\frac{d x_{1 i}}{d t} & =\frac{x_{2 i}-x_{1 i} a_{x}}{v_{x}} \\
\frac{d x_{2 i}}{d t} & =-\frac{R_{i}^{2}}{I_{y i}} \frac{d F_{x i}}{d t}+\frac{R_{i}}{I_{y i}} \frac{d B_{i}}{d t}-\frac{d a_{x}}{d t}
\end{aligned}\right.
$$

From the above model, longitudinal velocity, longitudinal acceleration and its derivative may be seen as external varying parameters. In this model, the equation relating to the coupling between the acceleration and the tyre force is not visible, but this choice of model does not imply that the coupling between acceleration and forces is neglected.

Remark 1: The paper focuses on the control of longitudinal front and rear slips under the assumption that the longitudinal velocity is available (measured or estimated). If only one wheel is used for braking, the vehicle speed may be estimated from the other wheel as it is done for traction [16]. However, if braking is done on the two wheels, speed estimation for motorcycles remains an open problem. Some recent works focus on the estimation of vehicles' speed like [17]. Throughout the paper, longitudinal velocity is assumed available.

\section{B. Tire dynamics}

Before detailing the tires' dynamics, let us make some assumptions:

Assumption 2:

- The road adherence is considered piecewise constant.

- The drag, lift and pitch aerodynamic forces are neglected.

- The time-derivative of the longitudinal acceleration is supposed known. Note that if the longitudinal acceleration is measured, it is easy to estimate its derivative even if the longitudinal acceleration is noised thanks to new differentiation methods such as the super-twisting algorithm [18].

- The motorcycle can be subject to a lateral motion but the velocity of the lateral slip angle must be bounded (i.e. the lateral slip angle must be of class $\mathcal{C}^{1}$ ).

The longitudinal tire forces are often considered proportional to the vertical forces [19]. Then, the longitudinal forces are modeled as follow:

$$
F_{x i}=F_{z i} \mu_{i}\left(\lambda_{i}, \alpha_{i}, \beta\right)
$$


where $\beta$ is the adherence of the road. The function $\mu_{i}\left(\lambda_{i}, \alpha_{i}, \beta\right)$ is called longitudinal friction coefficient. It depends on longitudinal slip $\lambda_{i}$, lateral slip $\alpha_{i}$ and road adherence $\beta$. Several mathematical formulas exist describing the longitudinal friction coefficient [19].

Form the assumption 2, the time derivative of the longitudinal forces is given by the following:

$$
\left\{\begin{aligned}
\frac{d F_{x i}}{d t} & =\frac{\partial F_{x i}}{\partial \lambda_{i}} \frac{d \lambda_{i}}{d t}+\frac{\partial F_{x i}}{\partial \alpha_{i}} \frac{d \alpha_{i}}{d t}+\frac{\partial F_{F_{i}}}{\partial F_{z i}} \frac{d F_{z i}}{d t} \\
& =F_{z i} \mu_{i}^{\prime}\left(\lambda_{i}, \alpha_{i}, \beta\right)+\mu_{i}\left(\lambda_{i}, \alpha_{i}, \beta\right) \frac{d F_{z i}}{d t}
\end{aligned}\right.
$$

where:

$$
\mu_{i}^{\prime}\left(\lambda_{i}, \alpha_{i}, \beta\right)=\frac{\partial \mu_{i}\left(\lambda_{i}, \alpha_{i}, \beta\right)}{\partial \lambda_{i}}\left(\frac{x_{2 i}-x_{1 i} a_{x}}{v_{x}}\right)+\frac{\partial \mu_{i}\left(\lambda_{i}, \alpha_{i}, \beta\right)}{\partial \alpha_{i}} \frac{d \alpha_{i}}{d t}
$$

Thanks to the assumption 2, the vertical forces are expressed by the following expressions [19]:

$$
\left\{\begin{array}{l}
F_{z f}=\frac{M\left(l_{r} g-h a_{x}\right)}{l_{f}+l_{r}} \\
F_{z r}=\frac{M\left(l_{f} g+h a_{x}\right)}{l_{f}+l_{r}}
\end{array}\right.
$$

where $l_{f}$ and $l_{r}$ are the distances between the motorcycle's center of mass and front and rear wheels respectively and $h$ is the height of the center of mass relative to the ground. Then:

$$
\frac{d F_{z i}}{d t}=(-1)^{j} \frac{M h}{l_{f}+l_{r}} \frac{d a_{x}}{d t}
$$

where $j=1$ if $i=f$ and $j=2$ if $i=r$.

\section{New time-scale model}

From equations (6) and (8), the second equation of the state space representation (4) is rewritten as:

$$
\frac{d x_{2 i}}{d t}=-c_{i}\left(F_{z i}\right) \mu_{i}^{\prime}\left(\lambda_{i}, \alpha_{i}, \beta\right)+\frac{R_{i}}{I_{y i}} \frac{d B i}{d t}-\frac{d a_{x}}{d t}\left((-1)^{j} \frac{R_{i}^{2}}{I_{y i}} \mu_{i}\left(\lambda_{i}, \alpha_{i}, \beta\right) \frac{M h}{l_{f}+l_{r}}+1\right)
$$

with: $c_{i}\left(F_{z i}\right)=\frac{R_{i}^{2}}{I_{y i}} F_{z i}$. By considering $U_{i}=\frac{R_{i} v_{x}}{I_{y i}} \frac{d B i}{d t}$, it follows:

$$
\frac{d x_{2 i}}{d t}=-c_{i}\left(F_{z i}\right) \mu_{i}^{\prime}\left(\lambda_{i}, \alpha_{i}, \beta\right)+\frac{U_{i}}{v_{x}}-\frac{\Delta_{i}}{v_{x}}
$$

where: $\Delta_{i}=v_{x} \frac{d a_{x}}{d t}\left((-1)^{j} \frac{R_{i}^{2}}{I_{y i}} \mu_{i}\left(\lambda_{i}, \alpha_{i}, \beta\right) \frac{M h}{l_{f}+l_{r}}+1\right)$.

The system (4) will be rewriten in a time scale as follows: $s(t)=\int_{0}^{t} \frac{d \tau}{v_{x}(\tau)}$ which implies that: $d t=v_{x} d s$. If one considers: $\dot{\chi}=\frac{d \chi}{d s}$, then: $\dot{\chi}=\frac{d \chi}{d t} \frac{d t}{d s}=\frac{d \chi}{d t} v_{x}$. The system (4) combined to the equation (10) gives:

$$
\left\{\begin{array}{l}
\dot{x}_{1 i}=x_{2 i}-x_{1 i} a_{x} \\
\dot{x}_{2 i}=-c_{i}\left(F_{z i}\right) \mu_{i}^{\prime}\left(\lambda_{i}, \alpha_{i}, \beta\right)+U_{i}-\Delta_{i}
\end{array}\right.
$$

This model takes into account the most important features of the longitudinal tires' dynamics: nonlinear longitudinal forces, load transfer, lateral slip angle and variation of the road adherence. The following section is devoted to the control of this system. In what follows, $\frac{d \chi}{d t}$ will refer to the time-derivative of $\chi$ and $\dot{\chi}=\frac{d \chi}{d s}$ to the derivative in the new time-scale. 


\section{CONTROLler DESIGN}

The present work aims to control motorcycle's longitudinal slip to a target one. This objective will be reached using a sliding mode controller. The synthesis methodology is almost the same if front or rear wheel braking is considered. In what follows, the braking is considered done on both wheels Firstly, a discussion about the choice of the sliding surface is given. Once done, a control law is proposed to control the state vector towards this sliding surface.

\section{A. Sliding surface}

Let us assume that the reference front and rear longitudinal slips are: $x_{1 f}^{*}$ and $x_{1 r}^{*}$. These reference slips are considered at least piece-wise constant. The following sliding surface is chosen:

$$
S_{i}=k_{i}\left(x_{1 i}-x_{1 i}^{*}\right)+\left(x_{2 i}-x_{2 i}^{*}\right)
$$

On the sliding surface, the system will be reduced to:

$$
x_{2 i}=x_{2 i}^{*}-k_{i}\left(x_{1 i}-x_{1 i}^{*}\right)
$$

and:

$$
\dot{x}_{1 i}=x_{2 i}^{*}-x_{1 i} a_{x}-k_{i}\left(x_{1 i}-x_{1 i}^{*}\right)
$$

If $x_{2 i}^{*}=a_{x} x_{1 i}^{*}$, the sliding surface becomes:

$$
S_{i}=k_{i}\left(x_{1 i}-x_{1 i}^{*}\right)+\left(x_{2 i}-a_{x} x_{1 i}^{*}\right)
$$

and the reduced system becomes:

$$
\dot{x}_{1 i}=-\left(k_{i}+a_{x}\right)\left(x_{1 i}-x_{1 i}^{*}\right)
$$

On the sliding surface with the reduced system, the longitudinal slip $x_{1 i}$ converges to the reference one $x_{1 i}^{*}$. The speed convergence of $x_{1 i}$ to $x_{1 i}^{*}$ depends on the constant gain $k_{i}$, the acceleration $a_{x}$ and the longitudinal velocity $v_{x}$ (because the system is expressed in a new time-scale). Indeed, to ensure a fast convergence of $x_{1 i}$ to $x_{1 i}^{*}$ on the sliding surface regardless of the longitudinal velocity and acceleration, the gain $k_{i}$ must be sufficiently large.

\section{B. Attractiveness of the sliding surface and control law}

Now, the objective is to control the sliding surface $S_{i}$ to zero in a finite time by means of the control $U_{i}$. From equations (11), (12) and (15), $\dot{x}_{1 i}$ can be rewritten as follows:

$$
\dot{x}_{1 i}=-\left(k_{i}+a_{x}\right)\left(x_{1 i}-x_{1 i}^{*}\right)+S_{i}
$$

Under the assumption that the longitudinal target slip is piece-wise constant, the dynamic of the sliding surface is given according the equations (11) and (17) by:

$$
\dot{S}_{i}=k_{i}\left[-\left(k_{i}+a_{x}\right)\left(x_{1 i}-x_{1 i}^{*}\right)+S_{i}\right]+U_{i}-\dot{a}_{x} x_{1 i}^{*}+\Delta_{i}^{\prime}
$$


where: $\Delta_{i}^{\prime}=-\Delta_{i}-c_{i}\left(F_{z i}\right) \mu_{i}^{\prime}\left(\lambda_{i}\right)$. Let us consider the following Lyapunov function:

$$
V_{i}=\frac{1}{2} S_{i}^{2}
$$

In order to achieve finite-time convergence of the system (18), the following condition should be satisfied:

$$
\frac{d V_{i}}{d t} \leq-\eta V^{\frac{1}{2}}, \eta>0
$$

which is equivalent to:

$$
\dot{V}_{i} \leq-\eta^{\prime} V^{\frac{1}{2}}, \eta^{\prime}=\eta v_{x}
$$

The time-derivative of $V_{i}$ in the new time-scale is computed as follows:

$$
\dot{V}_{i}=S_{i}\left(k_{i}\left[-\left(k_{i}+a_{x}\right)\left(x_{1 i}-x_{1 i}^{*}\right)+S_{i}\right]+U_{i}-\dot{a}_{x} x_{1 i}^{*}+\Delta_{i}^{\prime}\right)
$$

If the following control law is considered:

$$
U_{i}=-k_{i}\left[-\left(k_{i}+a_{x}\right)\left(x_{1 i}-x_{1 i}^{*}\right)+S_{i}\right]+\dot{a}_{x} x_{1 i}^{*}+v_{i}
$$

where $v_{i}$ is the forcing term and the other elements are the equivalent control law. The forcing term $v_{i}$ will be designed in order to counter the uncertain term $\Delta_{i}^{\prime}$.

From the equality (22) and the above control law, one obtains:

$$
\dot{V}_{i}=S_{i}\left(\Delta_{i}^{\prime}+v_{i}\right)
$$

Now, let us try to find an upper bound to $\left|\Delta_{i}^{\prime}\right|$.

- First of all, since $v_{x}$ is always positive and $\left|\mu_{i}\right|$ is always bounded by $\mu_{i, \max }$, one obtains:

$$
\left|\Delta_{i}\right|=v_{x}\left(\frac{R_{i}^{2}}{i_{y i}} \frac{M h}{l_{f}+l_{r}} \mu_{i, \max }+1\right)\left|\frac{d a_{x}}{d t}\right|
$$

- $F_{z i}$ is always bounded and one obtains: $\left|c_{i}\left(F_{z i}\right)\right| \leq \frac{R_{i}^{2}}{i_{y i}} M g$.

- Since $\mu_{i}\left(\lambda_{i}, \alpha_{i}, \beta\right)$ is Lipschitz with respect to $\lambda_{i}$ and $\alpha_{i}$ (see [19], [15]), $\left|\frac{\partial \mu_{i}\left(\lambda_{i}, \alpha_{i}, \beta\right)}{\partial \lambda_{i}}\right|$ can be bounded by $\mu_{1 i, \max }^{\prime}$ and $\left|\frac{\partial \mu_{i}\left(\lambda_{i}, \alpha_{i}, \beta\right)}{\partial \alpha_{i}}\right|$ by $\mu_{2 i, \max }^{\prime}$.

- Moreover, from the assumption 2, $\left|\frac{d \alpha_{i}}{d t}\right|$ can be bounded by $\alpha_{i, \max }^{\prime}$ which leads to:

$$
\left|c_{i}\left(F_{z i}\right) \mu_{i}^{\prime}\left(\lambda_{i}, \alpha_{i}, \beta\right)\right| \leq \frac{R_{i}^{2}}{i_{y i}} M g\left(\mu_{1 i, \max }^{\prime}\left|x_{2 i}-x_{1 i} a_{x}\right|+\mu_{1 i, \max }^{\prime} \alpha_{i, \max }^{\prime}\right)
$$

Consequently:

$$
\begin{aligned}
\left|\Delta_{i}^{\prime}\right| & \leq v_{x}\left(\frac{R_{i}^{2}}{i_{y i}} \frac{M h}{l_{f}+l_{r}} \mu_{i, \text { max }}+1\right)\left|\frac{d a_{x}}{d t}\right|+\frac{R_{i}^{2}}{i_{y i}} M g\left(\mu_{1 i, \text { max }}^{\prime}\left|x_{2 i}-x_{1 i} a_{x}\right|+\mu_{1 i, \text { max }}^{\prime} \alpha_{i, \text { max }}^{\prime}\right) \\
& =\left\|\Delta_{i}^{\prime}\right\|_{\infty}
\end{aligned}
$$

Thus, if $v_{i}$ is given by:

$$
v_{i}=-\left(\left\|\Delta_{i}^{\prime}\right\|_{\infty}+\sigma_{i}\right) \operatorname{sign}\left(S_{i}\right)
$$




$$
\begin{aligned}
& \text { with } \sigma_{i}>0 \text { and } \operatorname{sign}\left(S_{i}\right)=\left\{\begin{array}{ccc}
1 & \text { if } \quad S_{i} \geq 0 \\
-1 & \text { if } \quad S_{i}<0
\end{array}\right. \text {, it follows: } \\
& \dot{V}_{i} \leq-\sigma_{i}\left|S_{i}\right| \\
& \leq-\sqrt{2} \sigma_{i} V_{i}^{\frac{1}{2}}
\end{aligned}
$$

then, the control law $U_{i}$ that brings $S_{i}$ to zero in finite time is:

$$
U_{i}=-k_{i}\left[-\left(k_{i}+a_{x}\right)\left(x_{1 i}-x_{1 i}^{*}\right)+S_{i}\right]+\dot{a}_{x} x_{1 i}^{*}-\left(\left\|\Delta_{i}^{\prime}\right\|_{\infty}+\sigma_{i}\right) \operatorname{sign}\left(S_{i}\right)
$$

The constant gains $\sigma_{i}$ are set to adjust the finite time convergence of the sliding surfaces $S_{i}$ to zero. This gain must be carefully chosen. If it is chosen high enough, the controller will be robust to uncertainties $\Delta_{i}^{\prime}$; however, it can be conservative and induce a large control authority. So, a trade-off can be observed in the choice of the controller's gain in order to be robust and to avoid large control authority.

The finite time convergence $t_{r}$ of the sliding surface is obtained as follows: suppose that braking starts at $t=t_{0}$ and between $t_{0}$ and $t_{1}>t_{0}, x_{1 i}^{*}$ is constant, the longitudinal velocity for $t \in\left[t_{0}, t_{1}\right]$ should be less than $v_{x 0}=v_{x}\left(t_{0}\right)$ (because the vehicle is in a braking). Then, from the inequality (29), one obtains:

$$
\begin{aligned}
\frac{d V_{i}(t)}{d t} & \leq-\frac{\sqrt{2} \sigma_{i}}{v_{x}(t)} V_{i}^{\frac{1}{2}}(t) \\
& \leq-\frac{\sqrt{2} \sigma_{i}}{v_{x 0}} V_{i}^{\frac{1}{2}}(t)
\end{aligned}
$$

By integrating (31) over the time interval $t_{0} \leq \tau \leq t$ with $\left(t \leq t_{1}\right)$ :

$$
V_{i}^{\frac{1}{2}}(t) \leq-\frac{\sqrt{2} \sigma_{i}}{2 v_{x 0}} t+V_{i}^{\frac{1}{2}}\left(t_{0}\right)
$$

Consequently, $V_{i}(t)$ reaches zero in finite-time that is bounded by [20]:

$$
\begin{aligned}
t_{r} & \leq \frac{\sqrt{2} v_{x 0}}{\sigma_{i}} V_{i}^{\frac{1}{2}}\left(t_{0}\right) \\
& =\frac{v_{x 0}\left|S_{i}\left(t_{0}\right)\right|}{\sigma_{i}}
\end{aligned}
$$

Because $x_{1 i}^{*}$ is piece-wise constant (and not always constant), after each change of $x_{1 i}^{*}$ at instant $t_{c h}$, the sliding surface moves away zero and the new finite-time for the sliding surface to reach once again zero is:

$$
t_{r}-t_{c h} \leq \frac{v_{x}\left(t_{c h}\right)\left|S_{i}\left(t_{c h}\right)\right|}{\sigma_{i}}
$$

From the last equation, it can be seen that the finite-time for the sliding surface to converge to zero depends on the velocity of the vehicle. This should be taken into account to better choose the gain $\sigma_{i}$. Finally, the braking torque to apply, in standard time scaling, in order to control the longitudinal slip is given by:

$$
B_{i}=\int_{0}^{t} \frac{I_{y i}}{R_{i} v_{x}(\tau)} U_{i}(\tau) d \tau
$$

The proposed braking controller has been proved to be robust to longitudinal and vertical forces, road adherence and lateral slip angle. 


\section{SEEKING OF THE OPTIMAL LONGITUdinAL SLIP}

It has been said before that the longitudinal slip giving the maximal longitudinal force is very hard to find analytically. However, one can see that there exists a unique optimal longitudinal slip. So, a new method is proposed here to find this optimal slip empirically.

\section{A. Algorithm Perturb \& Observe (MPPT)}

The proposed algorithm is inspired from the Maximum Power Point Tracking (MPPT) methods used in the photovoltaic panels [21]. Among MPPT algorithms, the Perturb and Observe (P\&O) method is the most common for simplicity, ease of implementation, and good performance [21]. The principle of the Perturb \& Observe algorithm is the following.If the operating longitudinal slip is perturbed in a given direction and the longitudinal force for the corresponding wheel increases $\left(\frac{d F_{x i}}{d \lambda_{i}}>0\right)$, this means that the operating slip has moved towards the optimal one (see figure 1) and, therefore, the longitudinal slip must be further perturbed in the same direction. Otherwise, if the longitudinal force decreases $\left(\frac{d F_{x i}}{d \lambda_{i}}<0\right)$, the operating slip has moved away from the optimal one and, therefore, the direction of the slip perturbation must be reversed.

The Perturb \& Observe algorithm allows to find the longitudinal slip corresponding to the peak of the friction curve. However, if the road adherence is favorable, before the optimal longitudinal slip is reached, one can attempt the critical deceleration that causes the forward flip over of the motorcycle (figure 2). In this case, the previous algorithm must be turned off before reaching this critical deceleration.

Remark 2: Note that this algorithm requires knowledge of the longitudinal forces which is not obvious (see [14]). To overcome to this problem, two options are proposed:

- Consider only the front braking (which is sufficient in favorable road adherence). In this case and if the drag aerodynamic forces are neglected, the front longitudinal force can be approximated from the longitudinal acceleration by : $M a_{x}=F_{x f}$.

- If one wants to use both front and rear braking, the tires are considered having approximately the same optimal target slip. Both front and rear slips will be controlled to the same target slip. Moreover, the longitudinal acceleration is also used to approximate the sum of the longitudinal forces as follows: $M a_{x}=F_{x f}+F_{x r}$.

In this work, both front and rear braking are used. So, the second option is considered. The algorithm is resumed in the next section.

\section{B. Advanced algorithm for optimal longitudinal slip seeking}

The proposed algorithm is based on the following principles:

- The algorithm is triggered only when the rider requests a significant braking torque estimated as a hard braking. This can be detected by comparing the braking fluid pressure $\left(P_{b f}\right.$ and $\left.P_{b r}\right)$ to a maximal imposed one $\left(P^{*}\right)$. Moreover, to avoid chattering phenomenon when comparing the braking fluid pressures to the maximal one, a hysteresis function $f_{1}\left(P_{b i}\right)$ is used (see the following figure and the flowchart in figure 3 ). 
- If the algorithm is triggered and if the longitudinal deceleration of the motorcycle is less than the critical deceleration $\left(-a_{x}<-a_{x}^{*}\right)$, the $\mathrm{P} \& \mathrm{O}$ algorithm is started to find the optimal longitudinal slip corresponding to the peak of the friction curve.

- If the longitudinal deceleration is greater than or equal to the critical one, the $\mathrm{P} \& \mathrm{O}$ algorithm must be disabled and another algorithm is started in order to move away from the peak of the friction curve. In this case, the algorithm will perform the inverse task of the previous $\mathrm{P} \& \mathrm{O}$ algorithm.

- Ideally, this switching occurs when the longitudinal deceleration is equal to the critical one; in practice, the critical deceleration $-a_{x}^{*}$ is replaced with $-a_{x}^{*}-\delta_{a_{x}}$. This is important to guarantee a minimum amount of the vertical force on the rear wheel. Moreover, a hysteresis function $f_{2}\left(a_{x}\right)$ is also used here for the comparison $\left(\delta_{1}=1 \mathrm{~m} / \mathrm{s}^{2}\right.$ and $\left.\delta_{2}=2 \mathrm{~m} / \mathrm{s}^{2}\right)$ to avoid multiple switching between the two subprograms cited above (see the following figure and the flowchart in figure 3).

- The algorithm must be executed at a frequency relatively lower than the frequency of the controller, in order to satisfy the condition of $\lambda^{*}$ piece-wise constant, and also to allow the convergence of the longitudinal slip to the reference one before the next call of the optimizer algorithm.

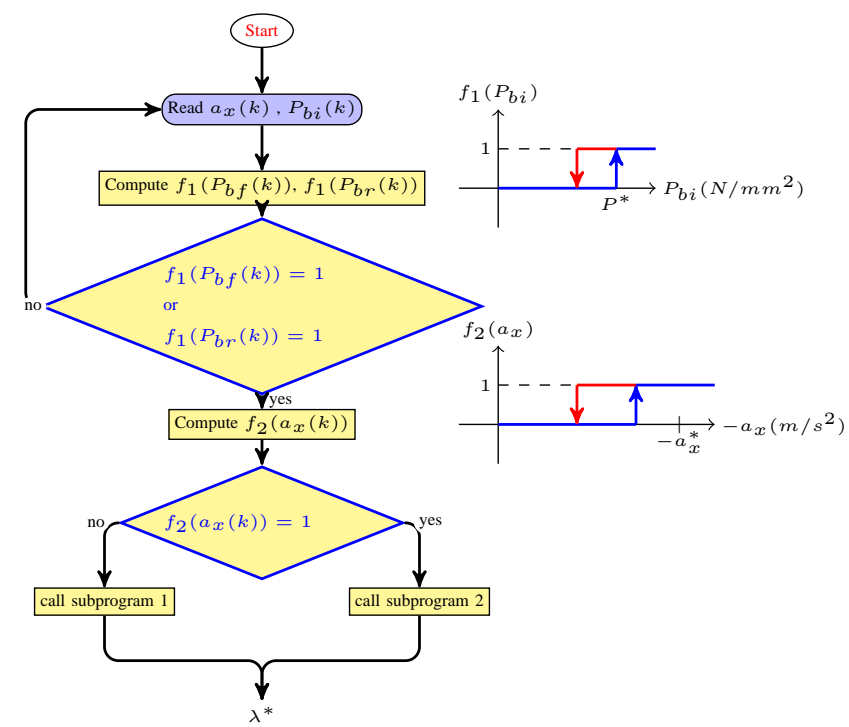

Fig. 3. Architecture of the whole program for the optimal braking

The architecture of the proposed algorithm is given in the figure 3. Because the longitudinal acceleration is used in this algorithm instead of the longitudinal forces, it is obvious that with the proposed algorithm, it is not possible to find the optimal front and rear longitudinal slips separately. So, only the longitudinal front slip will be used in the computation of the optimal slip. The choice of the front longitudinal slip is due to the fact that the front slip is more important in the braking phase (because of the load transfer).

The subprogram 1 is excuted in the case when the longitudinal deceleration is less than the critical one. In this case, the objective is to find the longitudinal slip which gives the maximum longitudinal force. Thus, the P\&O 
algorithm is called (figure 4 with the above red dashed rectangle). The subprogram 2 is called when there is a risk to reach the critical deceleration. In this case, the called subprogram will have the task to move the longitudinal slip away the maximum longitudinal force point and will perform the inverse task of the subprogram 1 . This is explained in the figure 4 with the bottom red dashed rectangle.

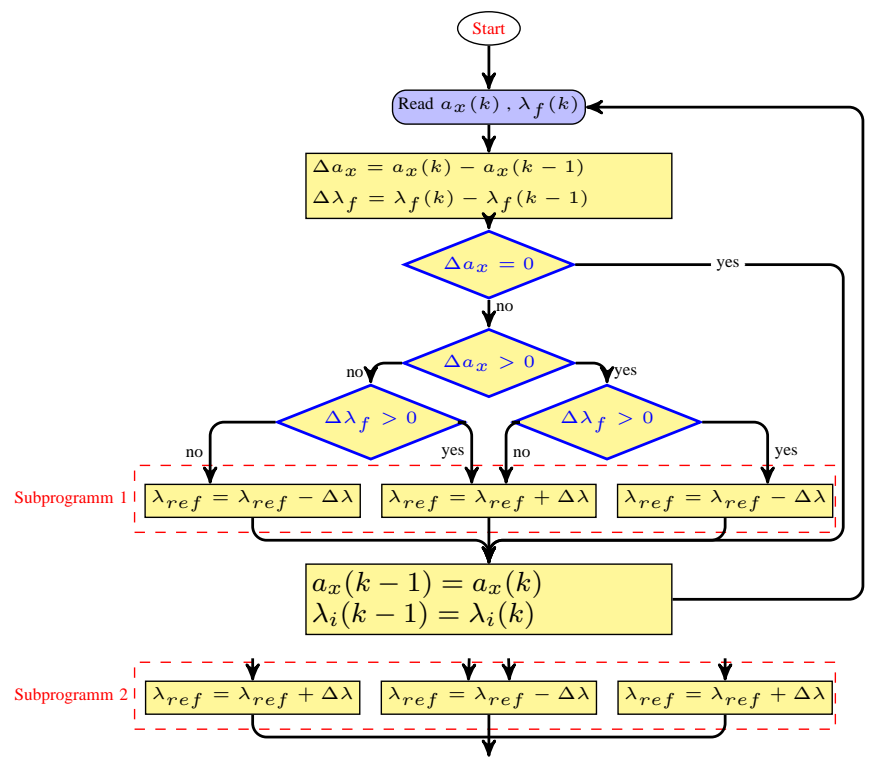

Fig. 4. Flowchart of the subprograms 1 and 2

Remark 3: The algorithm is not designed for the case when no wheel slip peak is present in the friction curve. In this case, the algorithm is supposed to converge towards the maximum target slip near - 1 , which corresponds to wheel lock. In this case, the algorithm may be saturated in order to avoid wheel lock.

\section{RESULTS OF SIMULATION}

To test the performances of the proposed controller, simulations are carried out on a motorcycle simulator (Mechanical Simulation Corp BikeSim) based on the AutoSim symbolic multi-body software [22], which takes into account all the motorcycle dynamics and the road-tires interaction forces [19]. Several scenarios are simulated to check the performances of the proposed controller. All the simulations are carried out with a sampling frequency of $2 \mathrm{kHz}$, which is available on motorcycle ECUs, except the optimizer algorithm which is executed at a lower frequency $5 \mathrm{~Hz}$. Moreover, in order to test the controller in the presence of measurement noise, $a_{x}$ and $\omega_{i}$ are assumed to be affected by a centered and random noise with magnitude $8 \%$ of the maximal values of the measured variables. For the longitudinal velocity, a more important error signal is considered to take into account noises and estimation uncertainties. It is assumed to be affected by a centered and random noise with magnitude $6 m^{-1} \mathrm{~s}^{-1}$.

In addition, braking fluid dynamics and actuator dynamics are taken into account in the simulations. Whole braking actuator is considered as a first order system with a time constant $t_{c}=0.061 \mathrm{~s}$. The following parameters are considered for the controller: $\alpha_{f}=\alpha_{r}=10^{3}, \sigma_{f}=2.10^{3}$ et $\sigma_{r}=10^{3}$. For the algorithm, the following 
parameters are considered: $P^{*}=4 \mathrm{MPa}$ and $\Delta \lambda=0.004$. For the time derivative of $a_{x}$, the super twisting algorithm is used [18].

\section{A. Dry asphalt and straight line}

First of all, a straight line and constant adherences about $\beta=0.85$ is considered. In this case, the road adherence is suitable and the load transfer may be important. Results of simulation of this scenario are depicted on figure 5 . In this scenario, the road adherence is favorable and it is easy to attempt the critical deceleration. This is why the subprogram 2 is often sought. From figure 5, the rear vertical force is positive when braking and a safety margin is always ensured to guarantee a minimum amount of the vertical force on the rear wheel. From simulation results, the braking torque to apply is reasonable and the chattering is not present thanks to the integral action in (35) and also to the fluid dynamics.
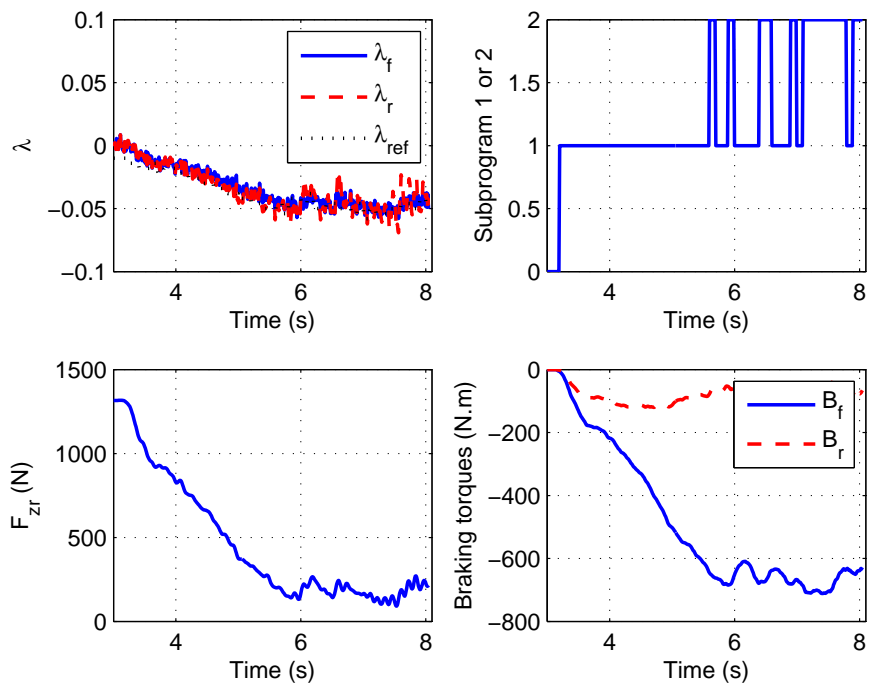

Fig. 5. $\beta=0.85$. (Top left) longitudinal front and rear slips and reference slip. (Top right) Mode of control: subprogram 1 or 2. (Bottom left) Vertical rear force. (Bottom right) Braking torques.

\section{B. Wet asphalt and straight line}

Now, a wet asphalt and a road adherence of $\beta=0.5$ are considered in a straight line. The associated results with the proposed controller are given in figure 6. Since the road adherence is low, it is difficult to attempt the critical deceleration. In this case, only the first subprogram will be sought. This can be seen from figure 6 . The proposed algorithm converge to a reference longitudinal slip of about -0.06 corresponding to the maximum longitudinal force slip point. Is this longitudinal slip the optimal one? Using the braking control law (35) and considering the target longitudinal slip constant, the fastest braking is obtained for $\lambda^{*}=-0.064$ with a braking distance of $172.40 \mathrm{~m}$. The 
braking distance when the optimizer algorithm is operating is $172.49 \mathrm{~m}$. This confirms that the proposed algorithm has find approximately the optimal target slip.
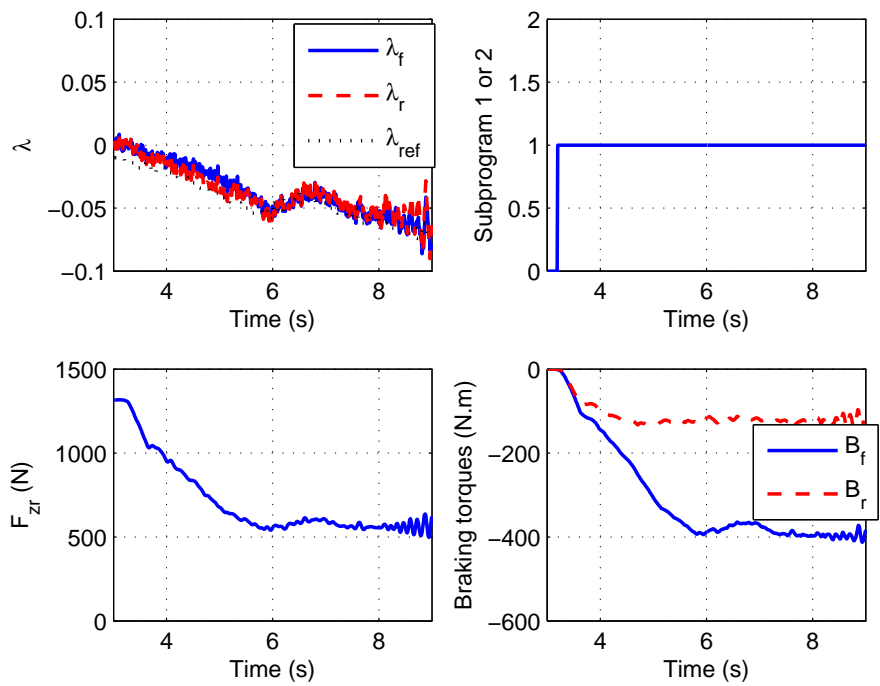

Fig. 6. $\beta=0.5$. (Top left) longitudinal front and rear slips and reference slip. (Top right) Mode of control: subprogram 1 or 2. (Bottom left) Vertical rear force. (Bottom right) Braking torques.

\section{Change of road adherence and straight line}

In the following scenario, a change of road adherence occurs when braking at $t=5.4 \mathrm{~s}$. Before this time, the road adherence was favorable $(\beta=1)$ and there was a risk of loss of contact of the rear wheel. This is why the subprogram 2 is sought and longitudinal slips converge to around $\lambda^{*}=-0.04$. From figure 7 , the rear vertical force is always greater than zero to avoid rear wheel's loss of contact. After $t=5.4 \mathrm{~s}$, the road adherence changes to $\beta=0.5$. Because of this change, the acceleration changes suddenly and in the same way the vertical forces. Thus, after $t=5.4 \mathrm{~s}$, only subprogram 1 will be sought. Moreover, form figure 7 , the robustness of the proposed controller to sudden changes in road adherence is also visible.

\section{Change of road adherence and cornering}

In the last case, a braking scenario is considered inside a curve with a change of road adherence at $t=3.8 \mathrm{~s}$ $(\beta=1 \underset{t=3.8 s}{\longrightarrow} 0.5)$. The results of simulation are given in figure 8 for the longitudinal dynamics and in the figure 9 for the lateral dynamics. From figure 8, the same previous remarks may be stated. Moreover, the robustness of the controller with respect to lateral movements is also shown. From the figure 9, the influence of the change in road adherence is visible at $t=3.8 \mathrm{~s}$ and the influence of braking is also visible from $t=5.2 \mathrm{~s}$. 

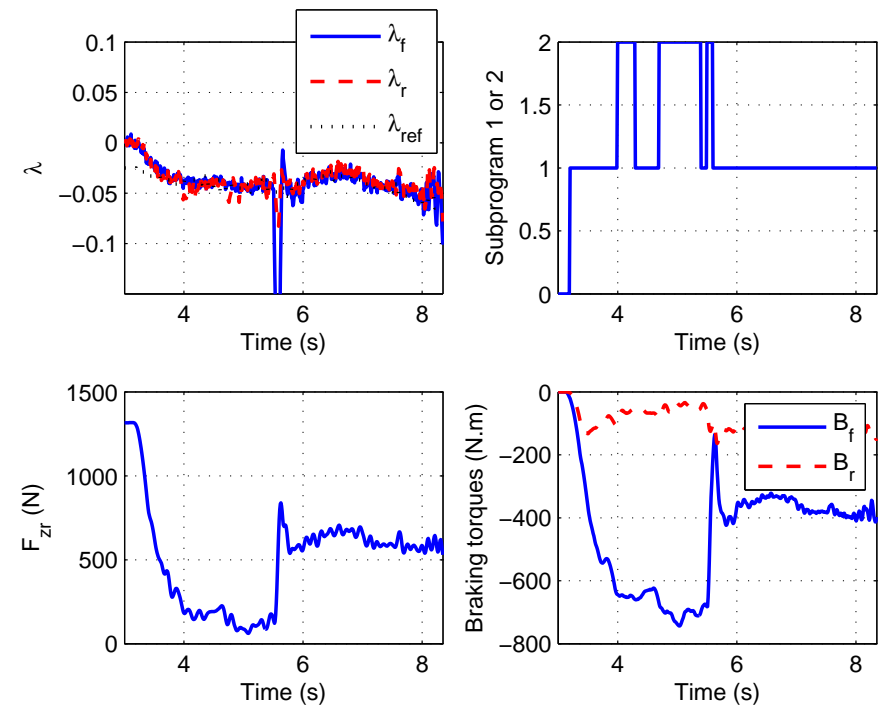

Fig. 7. $\beta$ varies from 1 to 0.5. (Top) longitudinal front and rear slips and reference slip from the algorithm. (Medium) Mode of control: subprogram 1 or 2. (Bottom) Vertical rear force.
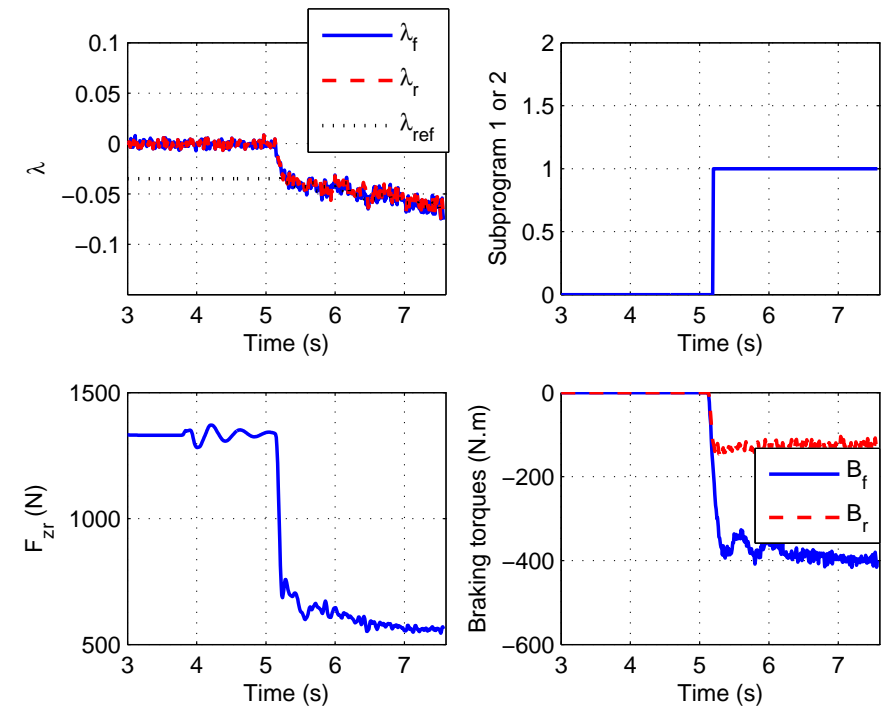

Fig. 8. Braking in a curve with low adherence. (Top) longitudinal front and rear slips and reference slip from the algorithm. (Medium) Mode of control: subprogram 1 or 2. (Bottom) Vertical rear force. 

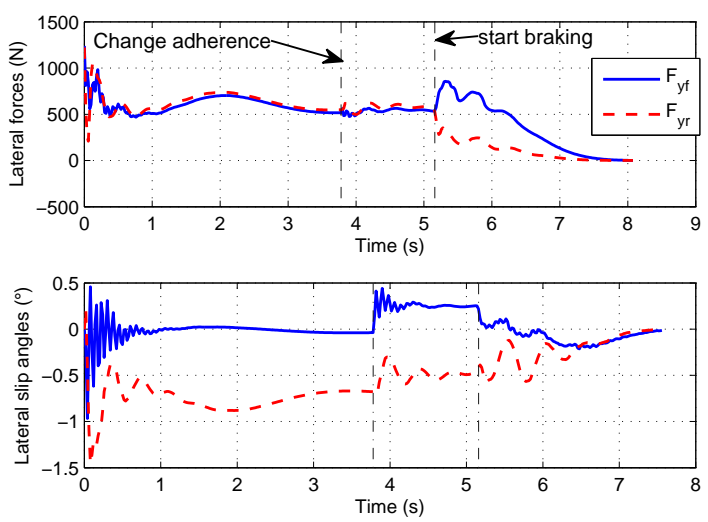

Fig. 9. Braking in a curve with low adherence. (Top) Lateral forces. (Bottom) Lateral slip angles.

\section{E. Discussions about the obtained results}

Finally, to better appreciate the performances of the proposed intelligent braking controller, the previous scenarios of braking are considered and the braking distances will be compared. For each scenario, comparison is done between the following cases:

- Using the braking control law (35) and the optimization flowchart in figure 3.

- Using the braking control law (35) and considering the target longitudinal slip constant $\left(\lambda^{*}=-0.02,-0.05\right.$, $-0.1,-0.2)$.

TABLE II

BRAKING DISTANCES

\begin{tabular}{|c|c|c|c|c|c|}
\hline$\lambda^{*}$ & Our algorithm & -0.02 & -0.05 & -0.1 & -0.2 \\
\hline$\beta=0.85$ & 130.44 & 215.60 & 129.14 & Stoppie & Stoppie \\
\hline$\beta=0.5$ & 172.49 & 238.27 & 174.36 & 176.93 & 196.35 \\
\hline$\beta=1 \underset{t=3.8 s}{\longrightarrow} 0.5$ & 139.69 & 227.76 & Stoppie & Stoppie & Stoppie \\
\hline$\beta=0.5$ and curve & 45.80 & 64.64 & 46.38 & 47.06 & Loss of control \\
\hline
\end{tabular}

The comparison is proposed in the table II. Using the sliding mode controller and considering the target slip constant, optimal braking (corresponding to low braking distance) is obtained for some scenarios when the constant target slip is about $\lambda^{*} \approx-0.05$. However, when the road adherence is acceptable, if the target slip is not well chosen, the rear wheel may lose contact with the ground (because of the load transfer) and an overturn of the motorcycle (stoppie) occurs. Moreover, in a curve, an inadequate choice of the target slip may cause skidding of the motorcycle. In the other cases when there is no danger, if the target slip is not well chosen, the fastest possible braking is not sure to be obtained. 
These remarks show that it is important to carefully choose the target slip which is often considered constant and known in classical slip controllers [12], [9]. This challenge was overcome with the proposed intelligent braking controller which automatically select the best target slip in order to avoid dangerous situations and provides approximately the fastest possible braking. Furthermore, both sliding mode controller and intelligent algorithm for the target slip seeking are robust to changes in road adherence.

\section{CONCLUSION}

As an alternative to conventional ABS systems, the proposed paper presents an innovative method to handle hard braking for motorcycles. First of all, a model for motorcycles tires and braking system was proposed and written in a new-time scale. After, the innovative braking system is proposed in two parts. First, an inner-loop braking controller is presented to track the longitudinal slip to a target one using a sliding mode controller. Second, an outer-loop algorithm is discussed to seek the best target slip in order to avoid dangerous situations and provides approximately the fastest possible braking. The performances of whole this innovative method are tested in the BikeSim multi-body simulator.

The future research will be devoted to:

- Resolve some limitations of the proposed work like the use of the longitudinal velocity which is not always measurable.

- Study in more details the influence of the lateral dynamics on the braking action and the influence of the braking on the lateral stability.

- The experimental validation of the control strategies.

\section{REFERENCES}

[1] M. Corno, M. Tanelli, S. M. Savaresi, L. Fabbri, and L. Nardo, "Electronic throttle control for ride-by-wire in sport motorcycles," in Control Applications, 2008. CCA 2008. IEEE International Conference on, 2008.

[2] M. E. H. Dabladji, D. Ichalal, H. Arioui, S. Mammar, and L. Fridman, "Estimation of lateral dynamics and road curvature for two-wheeled vehicles: A HOSM observer approach," in Proc. of the IFAC World Congress, 2014.

[3] A. T. Van Zanten, "Evolution of electronic control systems for improving the vehicle dynamic behavior," in Proceedings of the 6th International Symposium on Advanced Vehicle Control, 2002.

[4] S. Drakunov, U. Ozguner, P. Dix, and B. Ashrafi, "Abs control using optimum search via sliding modes," Control Systems Technology, IEEE Transactions on, vol. 3, no. 1, pp. 79-85, 1995.

[5] C.-M. Lin and C.-F. Hsu, "Self-learning fuzzy sliding-mode control for antilock braking systems," Control Systems Technology, IEEE Transactions on, vol. 11, no. 2, pp. 273-278, 2003.

[6] I. Petersen, T. A. Johansen, J. Kalkkuhl, and J. Lüdemann, "Wheel slip control in ABS brakes using gain scheduled constrained LQR," in European Control Conference, 2001.

[7] M. Corno, S. M. Savaresi, and G. J. Balas, "On linear-parameter-varying (lpv) slip-controller design for two-wheeled vehicles," International Journal of Robust and Nonlinear Control, vol. 19, no. 12, pp. 1313-1336, 2009.

[8] M. Tanelli, A. Astolfi, and S. M. Savaresi, "Robust nonlinear output feedback control for brake by wire control systems," Automatica, vol. 44, no. 4, pp. 1078-1087, 2008.

[9] W. Pasillas-Lpine, A. Lora, and M. Gerard, "Design and experimental validation of a nonlinear wheel slip control algorithm," Automatica, vol. 48, no. 8, pp. 1852 - 1859, August 2012. 
[10] M. Tanelli, C. Vecchio, M. Corno, A. Ferrara, and S. M. Savaresi, "Traction control for ride-by-wire sport motorcycles: A second-order sliding mode approach,” Industrial Electronics, IEEE Transactions on, vol. 56, no. 9, pp. 3347-3356, 2009.

[11] M. Tanelli, L. Piroddi, and S. Savaresi, "Real-time identification of tire-road friction conditions," Control Theory \& Applications, IET, vol. 3, no. 7, pp. 891-906, 2009.

[12] M. Corno, S. M. Savaresi, M. Tanelli, and L. Fabbri, "On optimal motorcycle braking," Control Engineering Practice, vol. 16, no. 6, pp. 644-657, 2008.

[13] M. Tanelli, M. Corno, I. Boniolo, and S. M. Savaresi, "Active braking control of two-wheeled vehicles on curves," International Journal of Vehicle Autonomous Systems, vol. 7, no. 3-4, pp. 243-269, 2009.

[14] M. E.-H. Dabladji, D. Ichalal, H. Arioui, and S. Mammar, "On the estimation of longitudinal dynamics of powered two-wheeled vehicles," in European Control Conference, 2015.

[15] V. Cossalter, Motorcycle Dynamics. Lulu. com, 2006.

[16] M. Corno, G. Panzani, and S. Savaresi, "Traction-control-oriented state estimation for motorcycles," Control Systems Technology, IEEE Transactions on, vol. 21, no. 6, pp. 2400-2407, Nov 2013.

[17] J. Villagra, B. D'Andréa-Novel, M. Fliess, and H. Mounier, "Estimation of longitudinal and lateral vehicle velocities: an algebraic approach," in 2008 American Control Conference, 2008.

[18] A. Levant, "Higher-order sliding modes, differentiation and output-feedback control," International journal of Control, vol. 76, no. 9-10, pp. 924-941, 2003.

[19] H. Pacejka, Tire and Vehicle Dynamics. SAE International, 2005.

[20] Y. Shtessel, C. Edwards, L. Fridman, and A. Levant, "Introduction: Intuitive theory of sliding mode control," in Sliding Mode Control and Observation, ser. Control Engineering. Springer New York, 2014, pp. 1-42.

[21] T. Esram and P. L. Chapman, "Comparison of photovoltaic array maximum power point tracking techniques," IEEE TRANSACTIONS ON ENERGY CONVERSION EC, vol. 22, no. 2, p. 439, 2007.

[22] R. Sharp, S. Evangelou, and D. Limebeer, "Advances in the modelling of motorcycle dynamics," Multibody System Dynamics, vol. 12, pp. 251 - 283, 2004. 